\title{
High-Resolution Pulse Shaper Based on Arrays of Digital Micromirrors
}

\author{
M. Knapczyk, A. Krishnan, L. Grave de Peralta, A. A. Bernussi, and H. Temkin
}

\begin{abstract}
We describe an optical Fourier transform pulse shaper based on a combination of a diffraction grating and a reconfigurable array of digital micromirrors. The pulse shaper allows for adjustment of separation between consecutive pulses within a pulse burst and the length of the burst. Pulse separation, burst length, and number of pulses can be dynamically reconfigured by changing the periodic pattern imposed to the array of digital micromirrors. Experimental results on binary intensity modulation are in excellent agreement with basic design relations describing the spectral and temporal characteristics of the pulse shaper.
\end{abstract}

Index Terms-Digital micromirrors, Fourier transforms, optical diffraction, optical pulses, optical pulse shaping.

G ENERATION of bursts of ultrafast optical pulses is critical to the implementation of optical code-division multiple-access [1] and optically assisted internet routing [2] networks. Reconfigurable Fourier transform pulse shapers (FT-PSs) based on liquid-crystal modulators (LCMs) and acustooptic modulators used as spatial masks have been demonstrated [3]-[6]. The use of microelectromechanical system devices with spatial resolution comparable to that of high-resolution LCMs has been also described for pulse-shaping applications [7] and for wavelength-division multiplexing [8], [9]. In this work, we demonstrate a novel approach to generate bursts of ultrashort pulses with dynamic control of the output sequence using an FT-PS based on a diffraction grating and a binary amplitude modulator formed by a digital micromirror array (DMA). DMAs developed for display applications offer high spatial resolution and are now commercially available [10]. Advantages of this approach include superior spatial resolution, established high reliability, fast reconfigurability and flexibility, control of the burst length, number of pulses, and pulse separation within the burst.

In the DMA used in this study, individual mirrors, fabricated over static random access memory address circuit, are suspended over an air gap by two postsupported torsion hinges at opposite corners. Depending on the control signal applied to the memory cell, each mirror can be rotated along its diagonal by $\pm 12^{\circ}$ from the unpowered position [10]. Each of the $\sim 750000$ mirrors of the rectangular chip can be addressed independently. The mirror switching time is less than $15 \mu \mathrm{s}$. In display ap-

Manuscript received May 18, 2005; revised June 16, 2005. This work was supported by Defense Advanced Research Projects Agency (DARPA) under a grant monitored by Dr. J. Shah, and by the J. F. Maddox Foundation.

The authors are with the Department of Electrical and Computer Engineering, Texas Tech University, Lubbock, TX 79409 USA (e-mail: maciej.knapczyk@ttu.edu).

Digital Object Identifier 10.1109/LPT.2005.854411

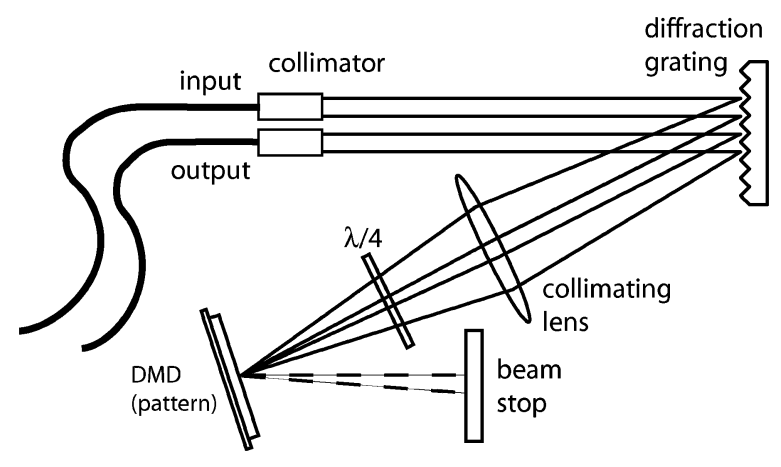

Fig. 1. Schematic diagram of the DMA-based FT-PS experimental setup.

plications, individual mirrors are reset at the video frame rate [11] in order to control hinge memory and stiction problems. In the pulse shaping experiments discussed here, the resets are disabled. Recent experiments in which mirror arrays were used to implement an all-optical switch [12] and a reconfigurable multiplexer [13] demonstrate excellent optical quality of these devices and their high mechanical stability.

In the experiments presented here, a passive mode-locked fiber laser generating 500 -fs pulses at the repetition rate of $50 \mathrm{MHz}$ and the center wavelength of $1560 \mathrm{~nm}$ was used as the input source (femtomaster laser from Fianium). The spectra were measured with an optical spectrum analyzer and temporal output profiles were obtained using free-space intensity autocorrelation. A schematic illustration of the experimental configuration of the DMA-based FT-PS is shown in Fig. 1. Input light is collimated onto a diffraction grating that disperses the frequency components at different angles. Our input beam is $\sim 5 \mathrm{~mm}$ in diameter and diffracted spectrum is focused on the surface of the DMA using the upper half of a lens with a focal length $(f)$ of $24 \mathrm{~cm}$. Approximately $20 \%$ of the surface of the DMA is illuminated, sufficient to cover the spectrum of the input laser. Selection of dispersed spectral components spatially distributed over the surface of the DMA is carried out by adjusting the ON-OFF state of lines of micromirrors. The DMA chip was rotated such that its miror diagonals coincided with the spectral dispersion direction. Selected frequency components are then reflected back, collimated onto the diffraction grating by the bottom half of the lens, recombined, and collected by the output collimator. A quarter-wave plate is included (see Fig. 1) to minimize the polarization dependence of the diffraction grating. The DMA-based pulse shaper exhibits polarization dependence loss of $0.75 \mathrm{~dB}$ and polarization-dependent wavelength shift of $0.03 \mathrm{~nm}$. 

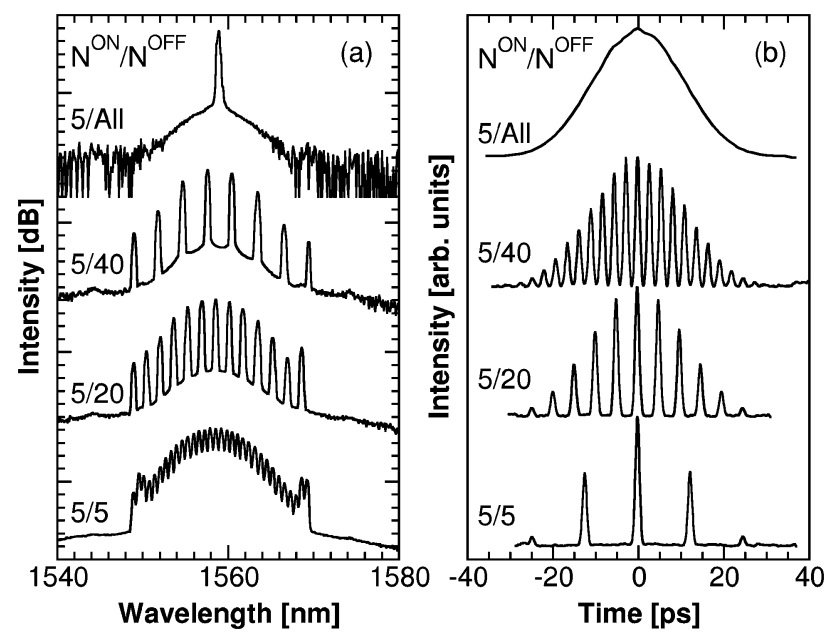

Fig. 2. Output (a) spectra and (b) intensity autocorrelation traces of the DMA-based pulse shaper obtained with different pattern periods $\left(N^{\mathrm{ON}}=5\right.$ and $N^{\mathrm{OFF}} \geq 5$ ). Vertical scale of the spectral plot (a) is $5 \mathrm{~dB} /$ division.

We use diffraction order $m=1$ of a grating with 600 lines/mm (grating period $d=1.6 \mu \mathrm{m}$ ) at nominal wavelength of $\lambda=1550 \mathrm{~nm}$. With an incident angle $\left(\theta_{\text {in }}\right)$ of $35^{\circ}$, the measured diffraction angle $\left(\theta_{d}\right)$ was $20.88^{\circ}$. The reciprocal of the spatial dispersion $(\alpha)$ of the grating-lens combination, which describes the relative position $(x)$ of different frequency components across the diagonal of the chip, is given by [4]

$$
\alpha=\frac{\partial \lambda}{\partial x}=\frac{d \cdot \cos \theta_{d}}{f \cdot m} .
$$

From (1), we estimate $\alpha \approx 6.49 \mathrm{~nm} / \mathrm{mm}$. Micromirrors of the DMA are $\sim 13 \times 13 \mu \mathrm{m}$ in size with $\mathrm{a} \sim 14-\mu \mathrm{m}$ pitch. Since the DMA miror diagonals are aligned with the spectral dispersion direction, the relevant distance between micromirror lines is given by the separation of their diagonals. The separation between adjacent micromirror lines $(D \approx 9.67 \mu \mathrm{m})$ is about equal to half of the micromirror diagonal. This results in spatial dispersion $\alpha \approx 0.063 \mathrm{~nm} /$ micromirror line.

The output spectrum of the pulse shaper is directly controlled by the DMA. When a periodic superstructure is imposed on the DMA, the output spectrum becomes multiple-peaked. Since the spectrum and the output temporal profile are related by a Fourier transform [4], multiple-peaked spectrum signifies a burst of pulses in the temporal domain. The repetition rate within the burst and the duration of the burst can be dynamically reconfigured by varying the periodic superstructure imposed on the DMA.

Fig. 2 shows the spectral and temporal output profiles of the pulse shaper for different sampling periods with five micromirror lines $\left(N^{\mathrm{ON}}=5\right)$ set to the reflecting state (ON-state) and a different number of micromirror lines set to the OFF-state $\left(N^{\mathrm{OFF}} \geq 5\right)$. When $N^{\mathrm{ON}}=5$ and all other micromirrors are set to the OFF-state, the output spectrum consists of a single peak [Fig. 2(a)]. This corresponds to a single elongated pulse in the temporal domain [Fig. 2(b)]. In contrast, sampling the DMA with different periods results in multiple peaks in the output spectra [Fig. 2(a)]. Sampling periods of 10, 25, and 45 micromirror lines results in peak-to-peak wavelength separation of $0.6,1.6$, and $2.8 \mathrm{~nm}$, respectively. The corresponding output
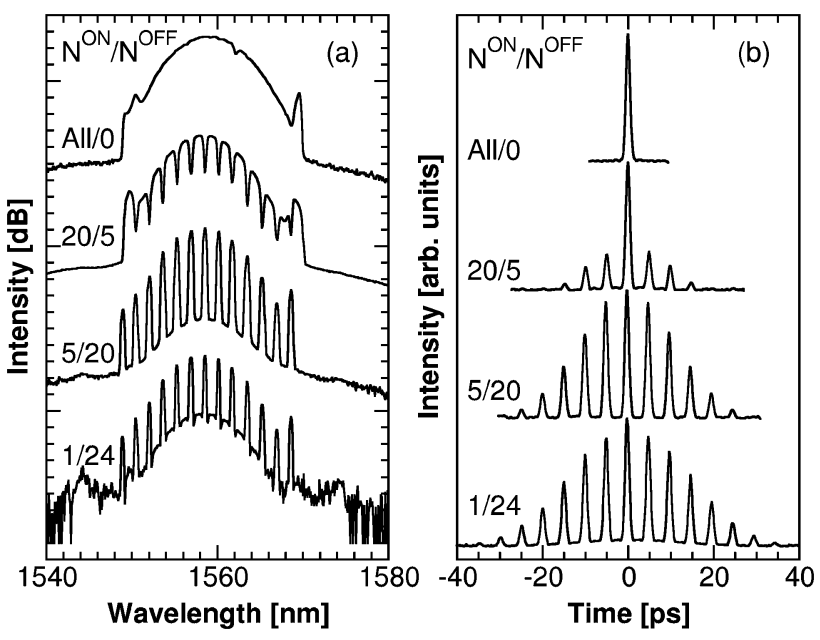

Fig. 3. Output (a) spectra and (b) intensity autocorrelation traces of the DMA-based pulse shaper obtained with constant pattern periods. The spectral and temporal output when all micromirrors are set to the ON-sate is also shown for comparison purposes. Vertical scale of the spectral plot (a) is $5 \mathrm{~dB} /$ division. The extinction ratio of $27 \mathrm{~dB}$ was reached for the $N^{\mathrm{ON}} / N^{\mathrm{OFF}}$ of 5/20.

pulse sequences for the same sampling periods are shown in Fig. 2(b). Periods of 10, 25, and 45 micromirror lines resulted in pulse separations of 12,5 , and $2.5 \mathrm{ps}$, respectively. As can be seen in Fig. 2(b), all pulse trains have the same length. This results from the same spectral width $(0.293 \mathrm{~nm})$ for all peaks in the spectra shown in Fig. 2(a).

The length of the output pulse sequence can be controlled by varying the width of individual peaks in the spectra. The burst length is inversely proportional to the spectral width of individual peaks [4] when the width corresponding to $N^{\mathrm{ON}}$ is larger than the diameter of the monochromatic beam spot $(\sim 80 \mu \mathrm{m}$ or nine micromirror lines) on the DMA. On the other hand, when $N^{\mathrm{ON}}<9$, the burst length varies inversely with respect to the peak spectral width, but is no longer inversely proportional. This is because the spectrum and the output temporal profile are related by a Fourier transform when the spot size can be considered a point. The spectra corresponding to the DMA sampled with a constant period $N^{\mathrm{ON}}+N^{\mathrm{OFF}}=25$ micromirror lines, where only $N^{\mathrm{ON}}$ is varied, are shown in Fig. 3(a). We also show the spectrum corresponding to all micromirrors set in the ON-state, a broad spectrum of the mode locked laser. When a periodic pattern is imposed to the DMA, the spectral width of the peaks is determined by the number of reflecting micromirror lines in the ON-state per period. Values of $N^{\mathrm{ON}}=1,5$, and 20 result in peak widths of $0.233,0.293$, and $1.155 \mathrm{~nm}$, respectively [Fig. 3(a)]. The corresponding intensity autocorrelation traces are shown in Fig. 3(b). When all micromirrors are set to the ON-state, the temporal output response consists of a single ultrashort pulse [see Fig. 3(b)]. Sampling the DMA with $N^{\mathrm{ON}}=1,5$, and 20 results in autocorrelation traces with lengths of 70, 50, and 30 ps, respectively. As shown in Fig. 3(b) all individual pulses within a burst have the same width independently of the period imposed on the DMA. The width of each output pulse within the burst is determined by the overall envelope of the output spectrum, which can be modified (not shown) by changing the number of periods in the DMA contributing to the output response. 

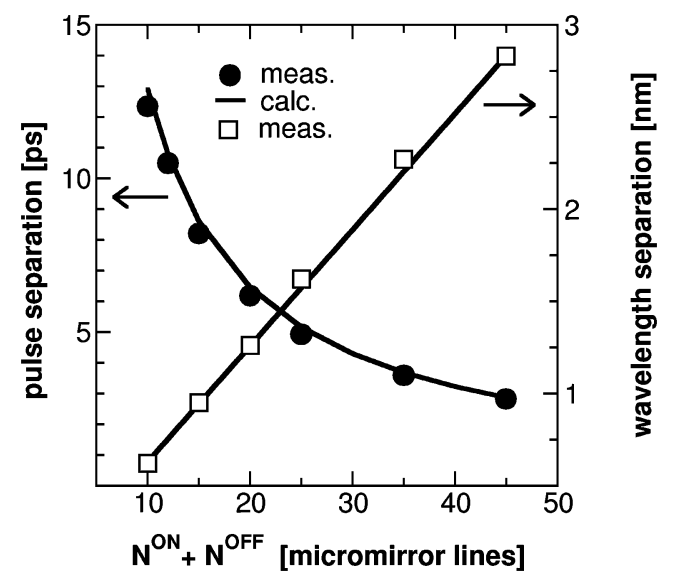

Fig. 4. Comparison between measured and calculated pulse separation and peak wavelength separation as a function of the DMA pattern period.

Since the spectrum and the output temporal profile of a pulse are related by a Fourier transform [4], the time separation $(\Delta \tau)$ between consecutive pulses in the burst and the peak-to-peak wavelength separation $(\Delta \lambda)$ in the spectrum are related by

$$
\Delta \tau=\frac{\lambda_{0}^{2}}{c} \cdot \Delta \lambda^{-1}
$$

where $\lambda_{0}$ is the center wavelength of the output spectrum and $c$ is the speed of light in free space. Using (1) and $\Delta x=D$. $\left(N^{\mathrm{ON}}+N^{\mathrm{OFF}}\right)$, we obtain an expression for $\Delta \lambda s$

$$
\Delta \lambda=\left(N^{\mathrm{ON}}+N^{\mathrm{OFF}}\right) \cdot D \cdot \alpha \cdot
$$

The pulse separation of the DMA-based FT-PS is determined from (2) and (3)

$$
\Delta \tau=\frac{\lambda_{0}^{2}}{c \cdot D \cdot \alpha} \cdot\left(N^{\mathrm{ON}}+N^{\mathrm{OFF}}\right)^{-1} .
$$

In order to verify the validity of our model, we show in Fig. 4 a comparison between the calculated and measured $\Delta \tau$ and $\Delta \lambda$ as a function of the sampling period. The time separation $\Delta \tau$ decreases inversely with the period $\left(N^{\mathrm{ON}}+N^{\mathrm{OFF}}\right)$, while the wavelength separation $\Delta \lambda$ increases linearly with the period $\left(N^{\mathrm{ON}}+N^{\mathrm{OFF}}\right)$, in excellent agreement with (4) and (3), respectively.

Equation (4) can be used to determine the DMA period required to obtain sequences of pulses with $1-\mathrm{THz}$ repetition rate within the burst. In our experiment, this corresponds to a pattern with a period of $N^{\mathrm{ON}}+N^{\mathrm{OFF}}=129$ micromirror lines. Measured intensity autocorrelation trace of the output response of the pulse shaper with this micromirror pattern is shown in Fig. 5. The output sequence consists of bursts with pulse separation of $1 \mathrm{ps}$, as calculated.

In summary, we demonstrate dynamic control of pulse sequence using an FT-PS that combines a diffraction grating and a reconfigurable array of digital micromirrors. When all the mirrors of the DMA are set to the ON-state, a single ultrashort pulse is observed at the output. A burst of pulses with a high repetition rate is obtained by imposing a periodic superstructure on

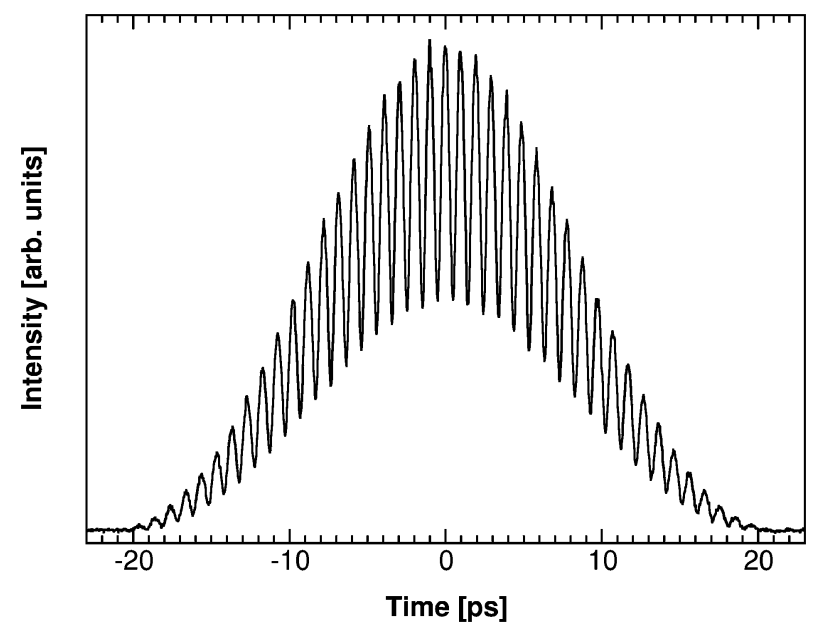

Fig. 5. Terahertz pulse train obtained with a pattern period of 129 micromirror lines.

the DMA. Sampling the DMA with different periods results in output sequences with variable pulse separation within the burst. The duration of the pulse burst can also be varied by changing the number of reflecting micromirrors in each period of the periodic superstructure formed on the DMA. Experimental results are in excellent agreement with basic design relations describing the spectral and temporal characteristics of the pulse shaper.

\section{REFERENCES}

[1] H. P. Sardesai, C. C. Chang, and A. M. Weiner, "A femtosecond code-division multiple-access communication system test-bed," $J$. Lightw. Technol., vol. 16, no. 11, pp. 1953-1964, Nov. 1998.

[2] M. C. Hauer, J. E. McGeehan, S. Kumar, J. D. Touch, J. Bannister, E. R. Lyons, C. H. Lin, A. A. Au, H. P. Lee, D. S. Starodubov, and A. E. Willner, "Optically assisted internet routing using arrays of novel dynamically reconfigurable FBG-based correlators," J. Lightw. Technol., vol. 21, no. 11, pp. 2765-2776, Jun. 2003.

[3] R. D. Nelson, D. E. Leaird, and A. M. Weiner, "Programmable polarization-independent spectral phase compensation and pulse shaping," Opt. Express, vol. 11, pp. 1763-1769, 2003.

[4] A. Weiner, "Femtosecond pulse shaping using spatial light modulators," Rev. Sci. Instrum., vol. 71, pp. 1929-1960, 2000.

[5] M. R. Fetterman, D. Goswami, D. Keusters, W. Yang, J.-K. Rhee, and W. S. Warren, "Ultrafast pulse shaping: Amplification and characterization," Opt. Express, vol. 3, pp. 366-375, 1998.

[6] G. Stobrawa, M. Hacker, T. Feurer, D. Zeidler, M. Motzkus, and F. Reichel, "A new high-resolution femtosecond pulse shaper," Appl. Phys. B, vol. 72, pp. 627-630, 2001.

[7] M. Hacker, G. Stobrawa, R. Sauerbrey, T. Buckup, M. Motzkus, M. Wildenhain, and A. Gehner, "Micromirror SLM for femtosecond pulse shaping in the ultraviolet," Appl. Phys. B, vol. 76, pp. 711-714, 2003.

[8] J. E. Ford and J. A. Walker, "Dynamic spectral power equalization using micro-opto-mechanics," IEEE Photon. Technol. Lett., vol. 10, no. 10, pp. 1440-1442, Oct. 1998.

[9] R. Ryf, Y. Su, L. Möller, S. Chandrasekhar, X. Liu, D. T. Neilson, and C. R. Giles, "Wavelength blocking filter with flexible data rates and channel spacing," J. Lightw. Technol., vol. 23, no. 1, pp. 54-61, Jan. 2005.

[10] M. A. Magnardi, "From ICs to DMDs," TI Tech. J., vol. 15, pp. 56-63, 1998.

[11] H. Chu, A. Gonzalez, T. Oudal, R. Alridge, D. Dudasko, and P. Barker, "DMD superstructure characterization," TI Tech. J., vol. 15, pp. 75-86, 1998.

[12] S. Sundaram, M. Knapczyk, and H. Temkin, "All-optical switch based on digital micromirrors," IEEE Photon. Technol. Lett., vol. 16, no. 6, pp. 807-809, Jun. 2003.

[13] A. A. Bernussi, L. Grave de Peralta, M. Knapczyk, R. Gale, and H. Temkin, "Reconfigurable sampling of the electric field at the reflecting surface of folded arrayed waveguide grating multiplexers," IEEE Photon. Technol. Lett., vol. 16, no. 10, pp. 2257-2259, Oct. 2004. 